\title{
Homology modelling and analysis of structure predictions of human tumour necrosis factor ligand superfamily member 8
}

Babatunde Joseph Oso ${ }^{1 *}$, Emmanuel Bukoye Oyewo ${ }^{2^{*}}$ and Adenike Temidayo Oladiji ${ }^{3}$

\begin{abstract}
Background: Tumour necrosis factor ligand superfamily member 8 (TNFL8) is a cytokine that plays vital roles in immune activations and inflammatory responses through its interaction with the tumour necrosis factor superfamily member. Despite multiple studies on the involvement of its receptor in the inflammatory response, there is limited information on the molecular characterization and structural elucidation of the cytokine. Considering the significance of the cytokine, the three-dimensional structure of TNFL8 model was generated by homology modelling through the Iterative Threading ASSEmbly Refinement (I-TASSER) server and validated through PROCHECK and Qualitative Model Energy Analysis (QMEAN) servers.

Results: The predicted structure has $90.00 \%$ of residues in the most favoured region of the Ramachandran plot while the QMEAN value gives - 3.06. The sequence and structural alignment between the generated model of the cytokine and template (1XU2) reveal that similar active site residues such as ILE-142, THR-175, GLU-178, and PHE-228 could be involved in binding pocket formation. However, docking studies of the 3D model of TNFL8 with eight phytochemicals from the extract of Xylopia aethiopica (Dunal) A. Rich revealed the phytochemical bound to two different binding sites which could be the active regions of the cytokine that could be essential for inhibition. More so, the docking analysis showed most of the phytochemicals have good binding affinity to the cytokine with ellagic acid showing the highest affinity with a binding energy of $-6.58 \pm 0.18 \mathrm{kcal} / \mathrm{mol}$.
\end{abstract}

Conclusion: The proposed model may shed light on the mechanisms of TNFL8 binding and provide insights into the identification of potential molecular targets for the development of the novel compound for the regulation of the functional activities of the cytokine.

Keywords: TNFL8, Homology modelling, I-TASSER, Molecular docking, Xylopia aethiopica (Dunal) A. Rich

\footnotetext{
*Correspondence: basjoe08@gmail.com; askafterbukoye@gmail.com

'Department of Biochemistry, McPherson University, Seriki Sotayo, Ogun

State, Nigeria

${ }^{2}$ Department of Biochemistry, Ladoke Akintola University of Technology,

Ogbomoso, Oyo State, Nigeria

Full list of author information is available at the end of the article
}

\section{Springer Open}

(c) The Author(s). 2021 Open Access This article is licensed under a Creative Commons Attribution 4.0 International License, which permits use, sharing, adaptation, distribution and reproduction in any medium or format, as long as you give appropriate credit to the original author(s) and the source, provide a link to the Creative Commons licence, and indicate if changes were made. The images or other third party material in this article are included in the article's Creative Commons licence, unless indicated otherwise in a credit line to the material. If material is not included in the article's Creative Commons licence and your intended use is not permitted by statutory regulation or exceeds the permitted use, you will need to obtain permission directly from the copyright holder. To view a copy of this licence, visit http://creativecommons.org/licenses/by/4.0/. 


\section{Background}

Tumour necrosis factor ligand superfamily member 8 (TNFL8) is a cytokine that plays modulatory roles on the biologic activities of tumour necrosis factor superfamily member 8 (TNFRSF8). Interaction of TNFL8 with cells expressing TNFRSF8 could induce cell proliferation or cell death [1]. TNFRSF8 (also known as CD30) is an inducible transmembrane receptor expressed on activated $\mathrm{T}$ cells, NK cells, B cells, and macrophages. It is also a known tumour marker usually expressed on many kinds of lymphoma and subsets of hyperactive T- and B-cell neoplasms $[2,3]$. The receptor contains an extracellular domain through which the TNFL8 binds and an intracellular domain responsible for mediation of various cellular responses, such as proliferation, differentiation, and apoptosis through recruitment of the TNFR-associated factor family of intracellular adapter molecules and subsequent activation of downstream protein kinase cascades and transcription factors such as the NF- $\mathrm{kB}$ and AP-1 family $[4,5]$. Tumour necrosis factor superfamily (TNFSF) ligands have been associated with costimulatory signals that regulate cell survival, cell proliferation, and cell differentiation. Thus, they play important roles in the pathogenesis of chronic inflammatory diseases such as atopic dermatitis, lupus erythematosus, rheumatoid arthritis, and asthma [6, 7]. Furthermore, TNFRSF8 is assumed to play an immunomodulatory role in the development of normal $\mathrm{T}$ cell through the elimination of autoreactive $\mathrm{T}$ cells and negative selection of $\mathrm{CD}^{+} / \mathrm{CD}^{+}$thymocytes [8]. Clinical significance of the cytokine has been associated with the monitoring of immune activations and elevated levels are found in patients with chronic infection [9]. Therapeutic compounds targeting the TNFRSF8/TNFL8 system have been reported to be effective therapies against the development of chronic inflammatory diseases [6]. Brentuximab vedotin (Fig. 1), an antibody drug conjugate consisting of combinations of a cytotoxic compound and a selective monoclonal antibody, is an example of such compounds. This agent has been used clinically for the treatment of various types of lymphoma [11].

Despite the assumed clinical importance of TNFL8, its molecular mechanism of action of the ligand is less understood. This could probably be related to slow progress in solving the TNFL8 structures. The knowledge of any protein structure underscores the understanding of the molecular functions of such a protein and its adaptation for drug design [12]. Over the past decades, computer-based methods have been developed for predicting the three-dimensional structure of a protein through algorithms to understand the details of a specific protein. These methods construct models based on the evolutionary relationship between the target sequence and a related template structure [13]. These computational tools are usually employed to predict the probable properties of proteins vis-à-vis the physicochemical, functional, and structural proprieties of the protein, and their interactions with biomolecule in a living cell [14]. Thus, the current study aimed at predicting the structural modelling and functional properties of TNFL8 as this could also enhance research into the modulatory mechanisms of TNFRSF8/TNFL8 interactions and signal mediation.

\section{Methods}

\section{Data retrieval and structural modelling}

The primary sequence of human tumour necrosis factor ligand superfamily member 8 was retrieved from the UniProt database (https://www.uniprot.org/) with UniProtKB ID P32971. SOPMA was used for the prediction of the cytokine's secondary structure (https://npsa-prabi. ibcp.fr/cgi-bin/npsa_automat.pl?page $=/$ NPSA/npsa sopma.html) [15]. The I-TASSER server (https:// zhanglab.ccmb.med.umich.edu/I-TASSER/) was used in modelling the tertiary structure $[16,17]$. The predicted model of the protein was refined through the GalaxyRefine web server (http://galaxy.seoklab.org/cgi-bin/submit. cgi?type=REFINE2) [18]. The structural characterization of the cytokine was carried out through the Ramachandran plot as obtained using the PROCHECK server (https://servicesn.mbi.ucla.edu/PROCHECK/) [19, 20] and also through the Qualitative Model Energy Analysis (QMEAN) using QMEAN Version 4.1.0 through the Swiss Model server (https://swissmodel.expasy.org/ qmean/) [21]. After validation of the 3D structure of the protein, further ligand-binding sites were analysed using COFACTOR (https://zhanglab.ccmb.med.umich.edu/ COFACTOR/) [22].

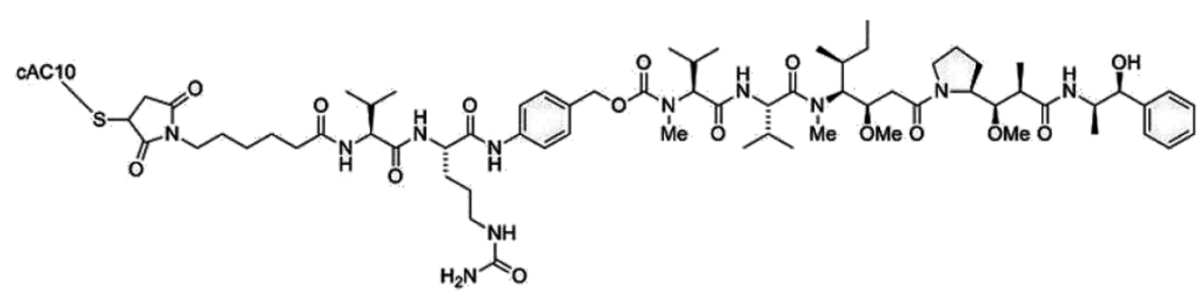

Fig. 1 Structure of brentuximab vedotin [10] 


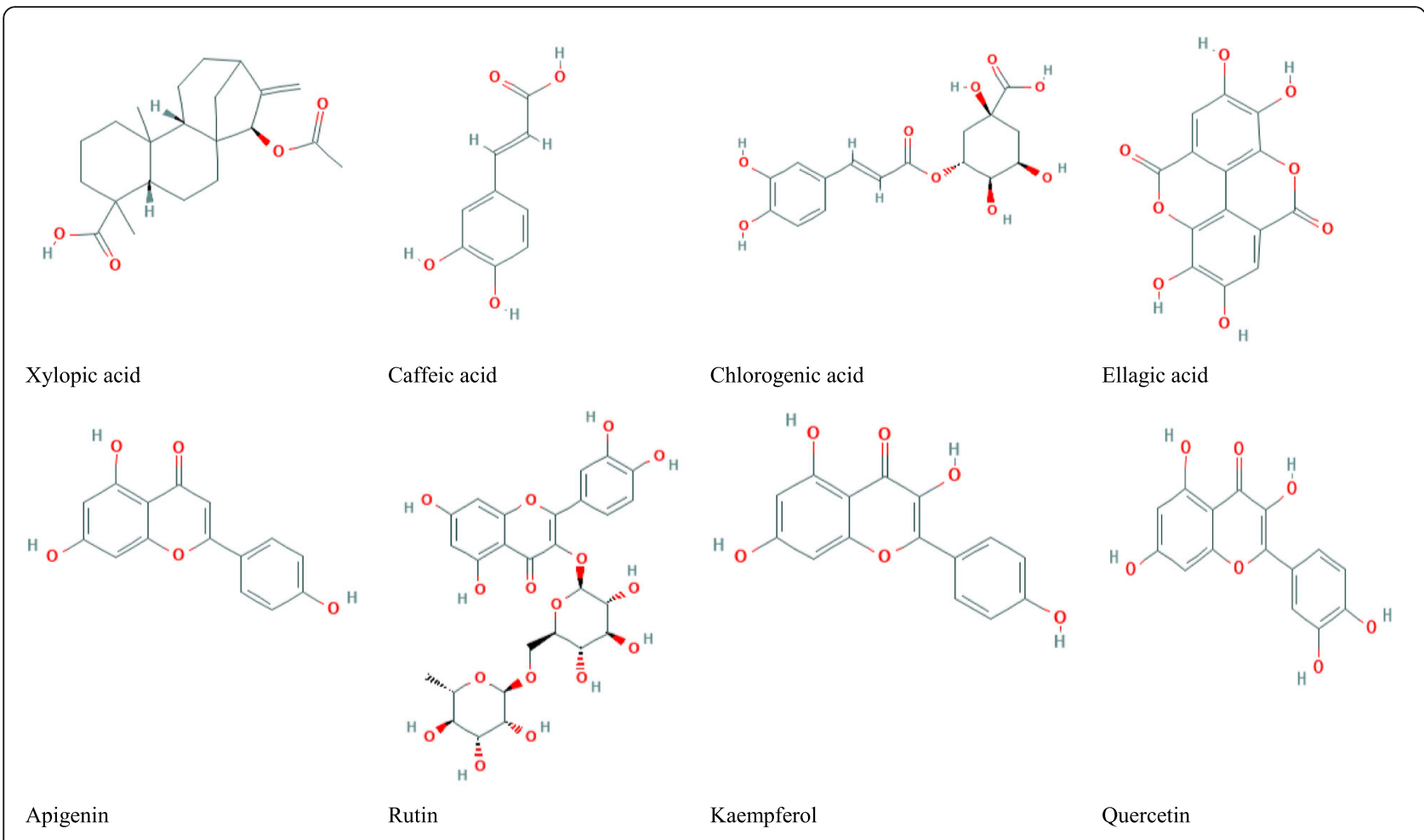

Fig. 2 Depiction of chemical structures of the selected compounds as obtained from the PubChem database

\section{Cytokine-ligand docking and molecular dynamics}

The structures of xylopic acid, caffeic acid, chlorogenic acid, ellagic acid, apigenin, rutin, kaempferol, and quercetin (PubChem CID: 354614, 689043, 1794427, 5281855, 5280443, 5280805, 5280863, and 5280343, respectively) were obtained from PubChem (https://pubchem.ncbi.nlm. nih.gov/) (Fig. 2) [23]. Open Babel was used to generate the pdbqt files [24]. Molecular docking studies were carried out in Auto Dock Vina [25]. The docking was performed within a restricted search space with centre set at $\mathrm{X}, 57.9893 ; \mathrm{Y}, 67.4664$; and Z. 61.7083 and dimension $\left(\mathrm{A}^{\circ}\right)$ set at X, 25.0000; Y, 25.0000; and Z, 25.0000. PyMOL ver. 1.1eval (De Lano Scientific LLC, CA, USA) was used for the visualization and graphical representations. The identification of non-covalent interactions between the model cytokine and the selected ligands was carried out by Protein-Ligand Interaction Profiler (https://projects.biotec. tu-dresden.de/plip-web/plip) [26]. The molecular dynamics simulations were carried out using the CABS-flex 2.0 server to evaluate the cytokine structural flexibility and stability of the cytokine-phytochemical complexes [27].
The root-mean-square fluctuations (RMSF) were obtained based on the MD trajectory or NMR ensemble with the default options.

\section{Results}

Data retrieval and structural modelling

The primary sequence of TNFL8 obtained from the UniProt database with accession no. P32971 and analysed through the ProtParam web server indicates that the cytokine contains 234 amino acids residues (Fig. 3). The analysis of the secondary structure was performed using SOPMA. The cytokine was predicted to exist in four states with $25.64 \%$ alpha-helix, $29.49 \%$ extended strand, $5.98 \%$ beta-turn, and $38.89 \%$ random coil (Fig. 4). The first five models predicted as 3D coordinate files from the I-TASSER server using the template sequences identified by LOMETS (Table 1) are presented in Fig. 5 with their respective c-scores. The resulted overall quality scores for all the models ranged from 41.6 to $50.7 \%$ as the percentage of the amino acid residues in the most favoured regions of the Ramachandran plot and - 18.03

Fig. 3 Primary sequence of TNFL8 


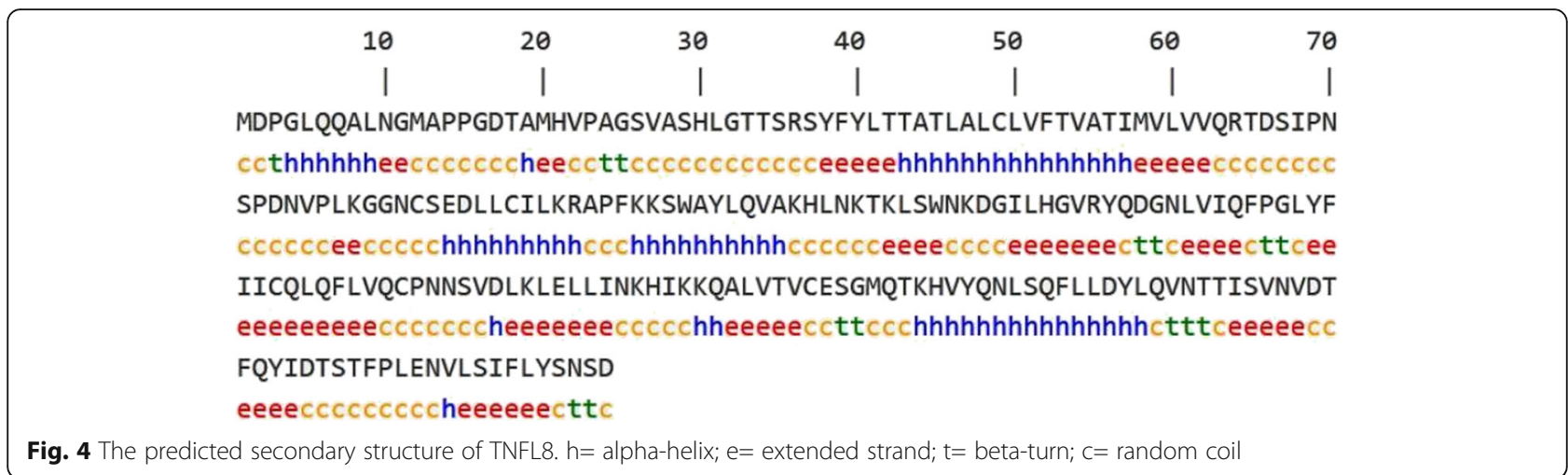

to - 14.42 QMEAN4 value (Fig. 6). The quality assessment of the selected model through the Ramachandran plot and QMEAN Z-scores obtained when refined through GalaxyRefine server showed $90.00 \%$ of the amino acids were under favoured region (red area), 8.6\% was under the allowed region (yellow area), and 1.4\% was observed under the disallowed region (Fig. 7) while the qualitative model energy analysis showed a QMEAN Z-scores value of - 3.06 (Fig. 8). The biological annotations of the cytokine based on the refined I-TASSER predicted structure deduced the ligand-binding site residues are ILE-142, THR-175, GLU-178, and PHE-228 as analysed via COFACTOR based on 1XU2 (the PDB Hit) as the template protein (Fig. 9).

\section{Cytokine-ligand docking and molecular dynamics}

The results of the respective binding energies and molecular interactions of the compounds identified from the fruit of $X$. aethiopica with the predicted model are

Table 1 Top 10 threading templates used by I-TASSER

\begin{tabular}{llllll}
\hline Rank & PDB Hit & Iden1 & Iden2 & Cov & Norm. Z-score \\
\hline 1 & $4 \mathrm{msvA}$ & 0.20 & 0.18 & 0.57 & 1.27 \\
2 & $4 \mathrm{msvA}$ & 0.19 & 0.18 & 0.56 & 1.91 \\
3 & $4 \mathrm{msvA}$ & 0.21 & 0.18 & 0.56 & 1.71 \\
4 & $3 i t 8$ & 0.22 & 0.15 & 0.59 & 2.77 \\
5 & 3it8 & 0.23 & 0.15 & 0.59 & 2.06 \\
6 & $4 \mathrm{msvA}$ & 0.19 & 0.18 & 0.56 & 1.76 \\
7 & 2re9 & 0.21 & 0.17 & 0.57 & 3.00 \\
8 & 2e7aA & 0.23 & 0.14 & 0.58 & 1.83 \\
9 & 6iy9A & 0.20 & 0.25 & 0.89 & 0.87 \\
10 & 2re9A & 0.22 & 0.17 & 0.56 & 3.75 \\
\hline
\end{tabular}

Ident1 percentage sequence identity of the templates in the threading aligned region with the query sequence, Ident2 percentage sequence identity of the whole template chains with query sequence, Cov the coverage of the threading alignment and is equal to the number of aligned residues divided by the length of query protein, Norm. Z-score normalized Z-score of the threading alignments. Alignment with a normalized Z-score $>1$ mean a good alignment and vice versa presented in Table 2 and Fig. 10. The results of the comparative analysis reveal that the binding affinity of the compounds ranged from $-5.22 \pm 0.26$ to $-6.58 \pm 0.18$ $\mathrm{kcal} / \mathrm{mol}$ with ellagic acid having the highest bindingfree energy followed by rutin $(-6.30 \pm 0.24 \mathrm{kcal} / \mathrm{mol})$, while caffeic acid had the least binding-free energy. The molecular illustrations of the interaction of the predicted model with the compounds are shown in Fig. 10 along with their corresponding interacting residues identified. The two noticeable binding sites were identified in the model structure through which the phytochemicals interacted with the ligand (Fig. 11). The investigations of interactions of the selected compounds with the ligand based on molecular dynamics are presented in Fig. 12. The RMSF graphs showed the flexibility of the amino acids were relatively low for the structures with bound phytochemicals compared to the wild-type TNFL8 without bound phytochemicals.

\section{Discussion}

The secondary structure reveals the recurring arrangements in space of adjacent amino acid residues in the cytokine. The proportion of the coil region demonstrates the stability of the protein [28]. The corresponding amino acid sequence was submitted to I-TASSER server to generate an ensemble of structural conformations when matched with template sequences identified by LOMETS, a meta-server containing multiple threading programs, from the PDB library (Table 1). The templates are experimentally determined protein structures with maximum similarity with the submitted sequence aligned through TM-align structural alignment program algorithms [16]. The templates of the highest significance in the threading alignments measured by the Zscore were used. The generated ensemble of structural conformations, called decoys, were clustered through the SPICKER program based on the pair-wise structure similarity to predict the top five models ranked based on their corresponding C-scores (Table 2, Fig. 5). The respective quality of the tertiary structures of the predicted 


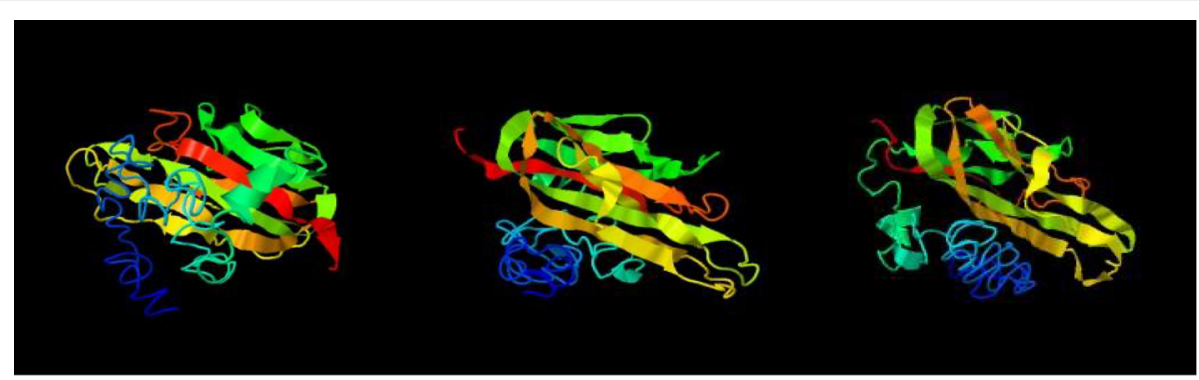

(1) $\mathrm{C}$-score $=-3.27$

(2) C-score $=-3.35$

(3) C-score $=-3.44$

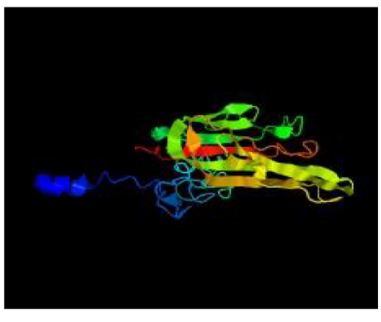

(4) $\mathrm{C}$-score $=-3.89$

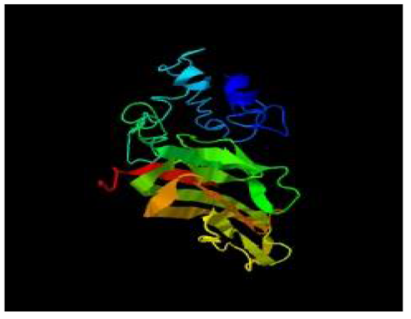

(5) $\mathrm{C}$-score $=-4.08$

Fig. 5 Top 5 final models predicted by I-TASSER (1) model 1, (2) model 2, (3) model 3, (4) model 4, and (5) model 5
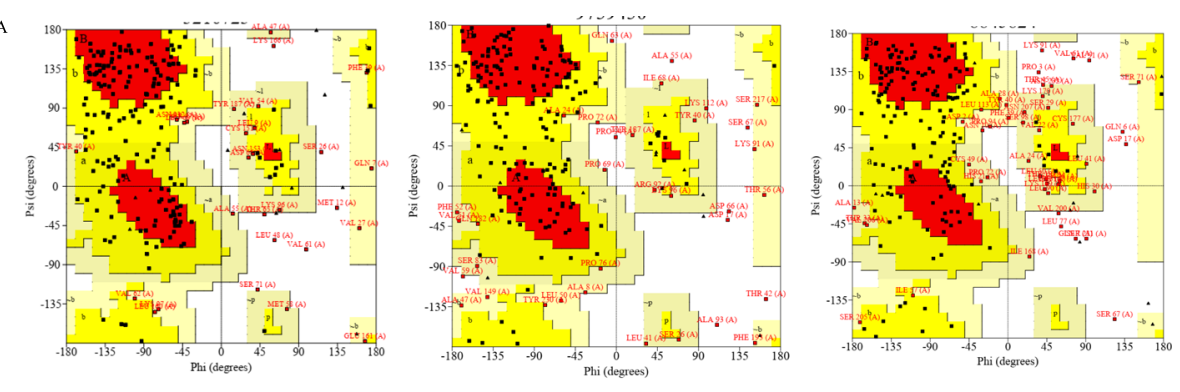

(1)

(2)

RMFR $=49.3 \%$

(3)

RMFR $=41.6 \%$
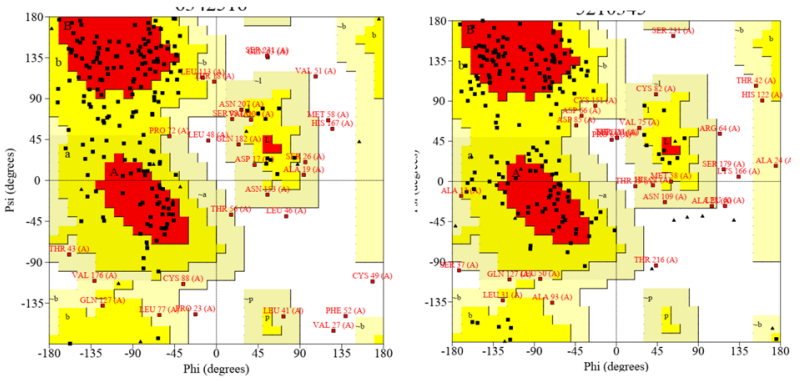

(4) $\mathrm{RMFR}=50.7 \%$

(5)

RMFR $=48.8 \%$

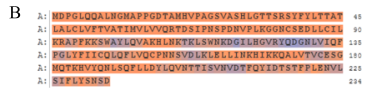

(1) QMEAN4 Value $=-16.50$

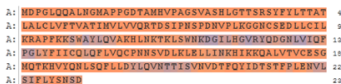

(2) QMEAN4 Value $=-18.03$

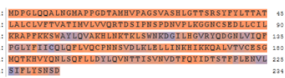

(3) QMEAN4 Value $=-17.72$

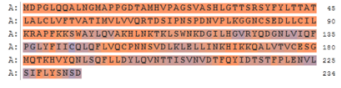

(4) QMEAN4 Value $=-14.42$

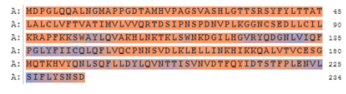

(5) QMEAN4 Value $=-15.24$

Fig. 6 Quality of (1) model 1, (2) model 2, (3) model 3, (4) model 4, and (5) model 5 predicted by I TASSER showing a the Ramachandran plots showing the percentage of the residues in most favoured regions (RMFR) and $\mathbf{b}$ QMEAN showing the sequence coloured by local quality with the colour gradient ranges from orange (low quality) to blue (high quality) and QMEAN4 value 


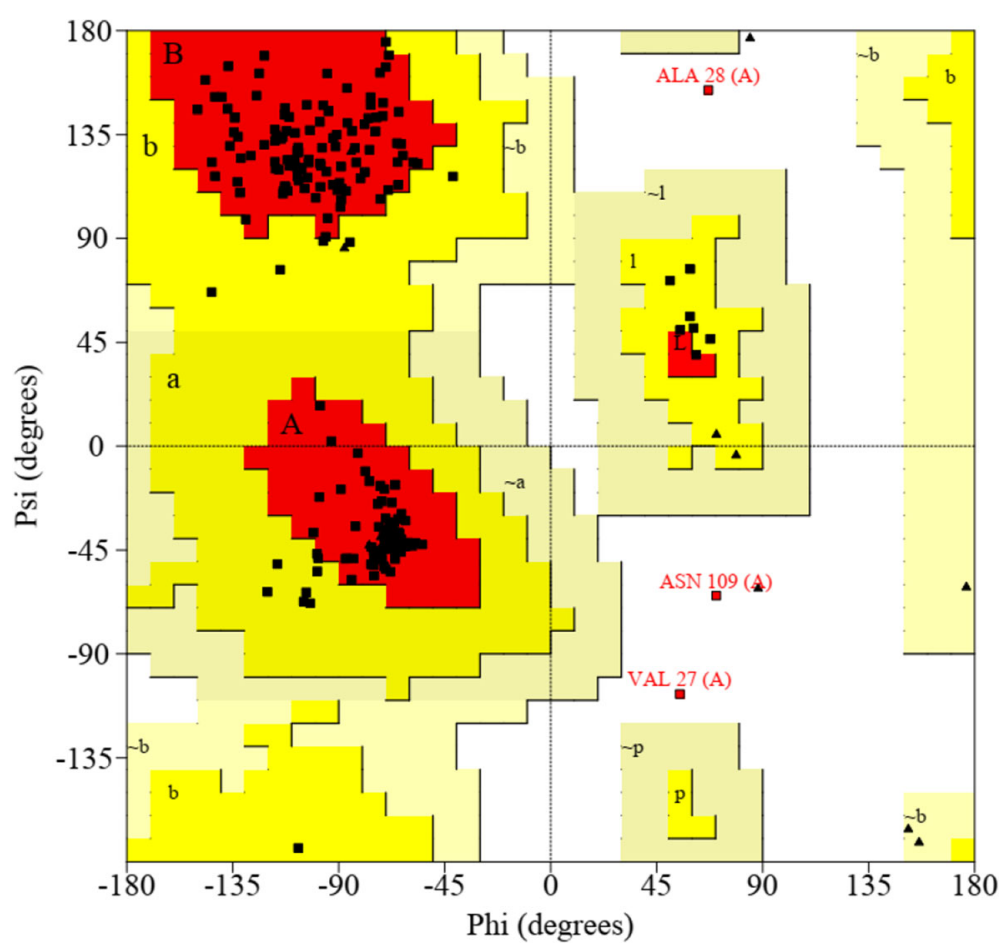

Fig. 7 Ramanchandran plot showing the residues in most favoured regions $[A, B, L]=188$, residues in additional allowed regions $[a, b, l, p]=18$, residues in generously allowed regions $[\sim a, \sim b, \sim 1, \sim p]=$ Nil, residues in disallowed regions $=3$, number of non-glycine and non-proline residues $=$ 209, number of end-residues (excl. Gly and Pro) $=2$, number of glycine residues (shown as triangles) $=12$, and number of proline residues $=11$

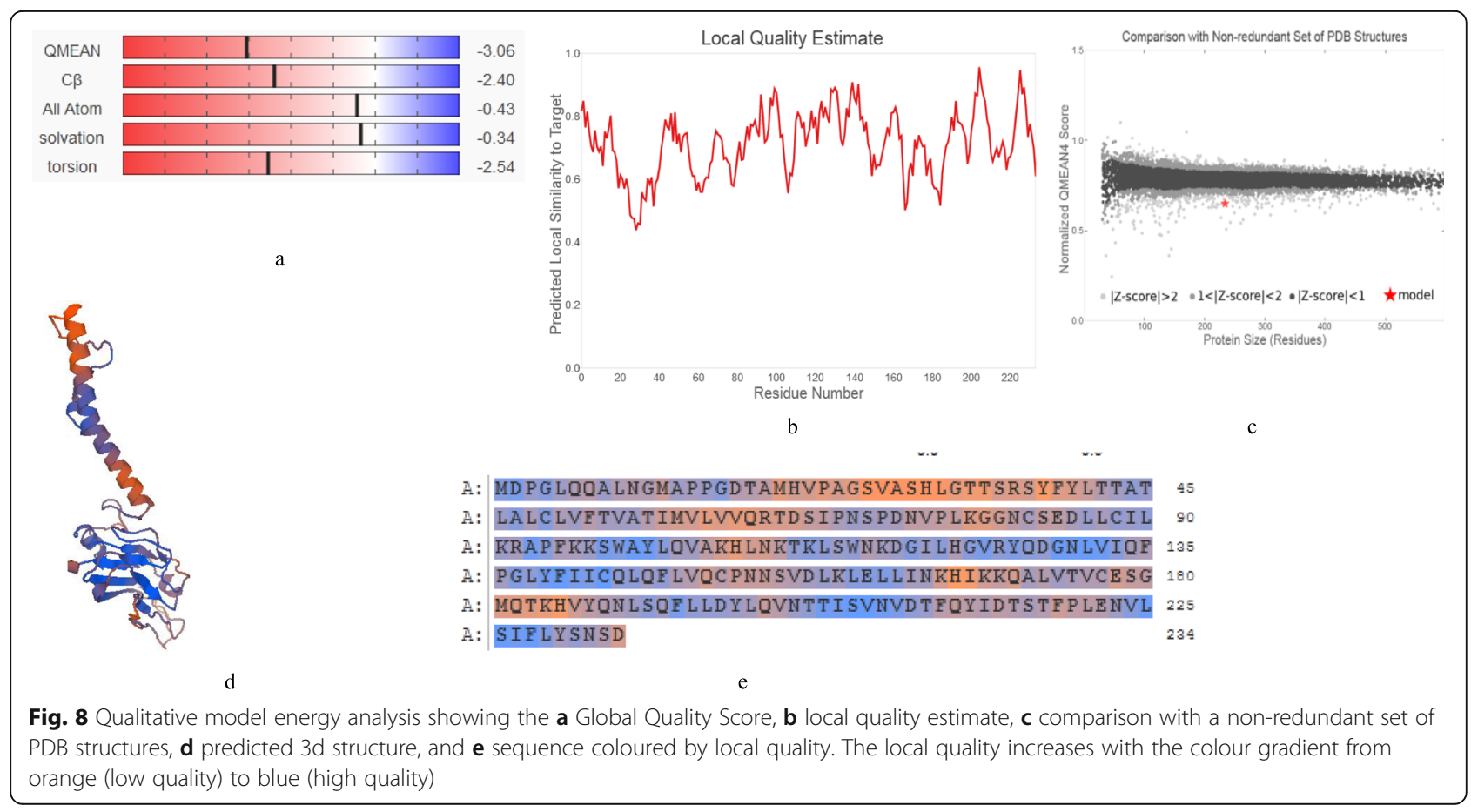




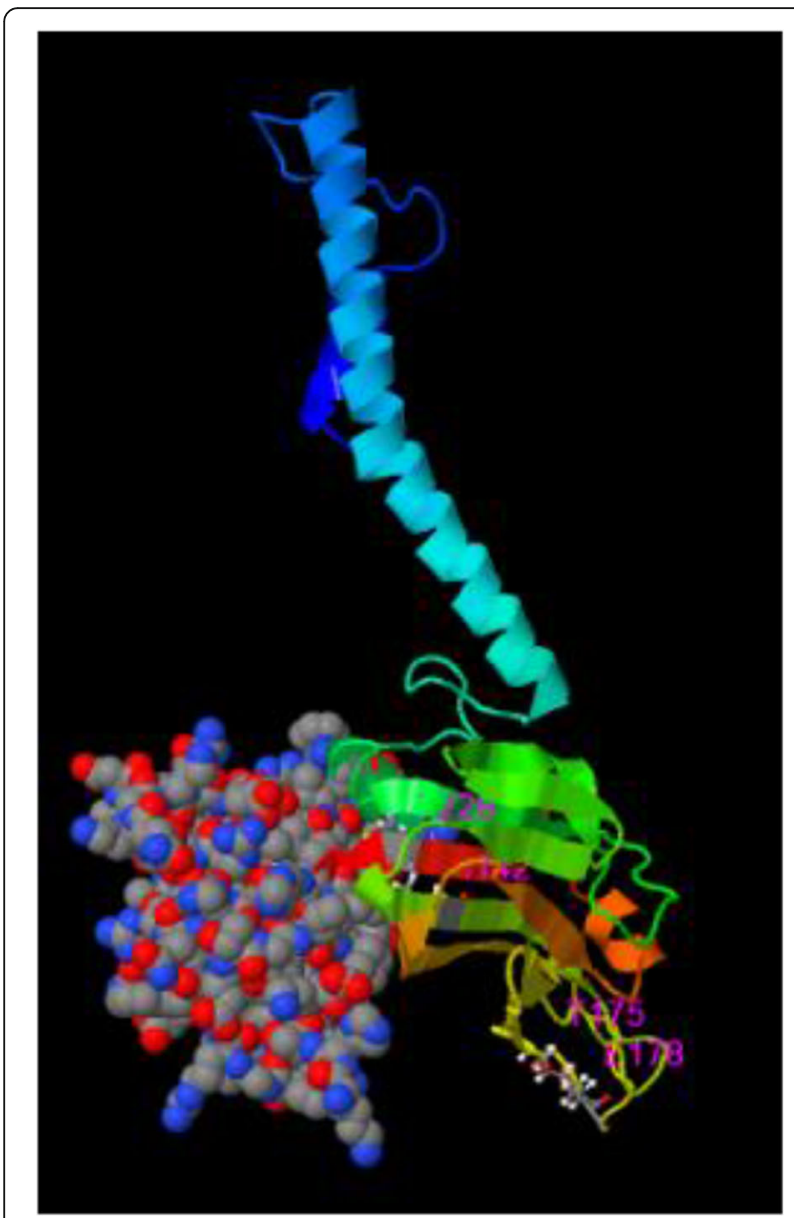

Fig. 9 Predicted ligand-binding sites

models from I-TASSER was assessed through the Ramachandran plots obtained from PROCHECK, a server that determines the stereochemical quality of protein structures and QMEAN, an accuracy prediction score [2022]. The obtained values were lower than the acceptable values of the respective analysis whereas an acceptable protein model would be predictable to have over $90 \%$ in the most the favoured regions in the Ramachandran plot based on an analysis of 118 structures of resolution of at least $2.0 \AA$ and the QMEAN4 value must be around zero and not below -4.0 as an indication of the degree of nativeness of the model to an experimental structure of similar size [21]. Thus, further refinements of the predicted models were performed to minimize the probable errors in the predicted structures via the GalaxyRefine2 server and ranked based on their respective Galaxy energies. The server adopts an iterative optimization approach for refining proteins and generates several models with more structural deviations from the submitted model. The iteratively refined models were further validated through Ramachandran plots obtained from PROCHECK and QMEAN [19-21]. The best model was selected objectively based on the quality of the protein structure. The Ramachandran plot obtained for the quality assessment of the selected model showed that 90.0\% of the structure was under favoured region (red area), $8.6 \%$ was under the allowed region (yellow area), and $1.4 \%$ was observed under the disallowed region, signalling a high quality of the predicted structure (Fig. 7). The amino acid residues in the disallowed region are VAL-27, ALA-28, and ASN-109. Additionally, the qualitative model energy analysis showed a QMEAN Z-scores value of -3.06 , which is an acceptable score as the value is greater than -4.0 (Fig. 8a) [21]. This indicates the homology model has a good agreement with the experimental structures of similar size. The predicted Z-score compares the interaction potential between $C \beta$ interaction energy, all atoms pair-wise energy, the solvation potential, and the torsion angle potential [29]. The estimate of the local quality (Fig. 8b) shows a larger proportion of the residues ( $x$-axis) of the model have high quality in comparison with the native structure (y-axis) with scores above 0.6 (Fig. 8c). The comparison with the non-redundant set of PDB structures relates the quality scores of the model with obtainable scores for experimental structures of similar size. This showed the model has a normalized QMEAN4 score within 1.0 and 2 of the standard deviation of the mean of $\mathrm{z}$ score (|Zscore|). This is also indicated by the sequence coloured by local quality (Fig. 8d, e).

Activation of TNFR in response to TNFL8 stimulation induces the recruitment of signalling proteins that mediate signal transduction events which are capable of eliciting stimulatory signals such as the production of the pro-inflammatory chemokine [30] Targeting TNFRSF8/ TNFL8 interactions has been reported to be a useful mechanism in the regulation of pathophysiologic roles of TNFRSF8/TNFL8 activities mostly in oncology and chronic inflammatory diseases [6]. Therapeutic antibodies targeting the TNF family members have been found to exert antagonist signalling effects on the physiologic functions of TNFRSF8 by blocking TNFR SF8/TNFL8 interactions. Moreover, therapeutic agents derived from natural sources have been reported to possess the ability to suppress the expression and signalling of TNF family members [31]. Furthermore, consumption of strawberry significantly decreased soluble TNFR in a randomized cross-over double-blind placebo-controlled trial [32]. The predicted binding sites (Fig. 9) identified through COFACTOR could be targets of potential substrates or inhibitor. The conformational analysis of the cytokine with ligands was simulated through molecular docking to analyse the molecular interaction of the bioactive compounds from Xylopia aethiopica (Dunal) A. Rich fruit with the selected model. The ligands selected were based on a previous report on the interaction of 
Table 2 Binding energy and non-covalent interaction of TNFL8 with the selected phytochemicals

\begin{tabular}{|c|c|c|c|c|c|c|c|}
\hline \multirow[t]{2}{*}{ Ligands } & \multirow{2}{*}{$\begin{array}{l}\text { PubChem } \\
\text { ID }\end{array}$} & \multirow{2}{*}{$\begin{array}{l}\text { Binding } \\
\text { energy } \\
\text { (kcal/mol) }\end{array}$} & \multicolumn{5}{|l|}{ Non-covalent interaction } \\
\hline & & & Hydrophobic interactions & Hydrogen bond & Salt bridges & $\pi$-Stacking & $\pi$-Cation interactions \\
\hline Xylopic acid & 354614 & $-5.76 \pm 0.39$ & $\begin{array}{l}\text { VAL-62 } \\
\text { PRO-69 }\end{array}$ & $\begin{array}{l}\text { THR-65 } \\
\text { ASP-66 }\end{array}$ & - & - & - \\
\hline Caffeic acid & 689043 & $-5.22 \pm 0.26$ & $\begin{array}{l}\text { PRO-69 } \\
\text { LYS-117 }\end{array}$ & ASN-70 & LYS-117 & - & - \\
\hline Chlorogenic acid & 1794427 & $-5.86 \pm 0.45$ & $\begin{array}{l}\text { ARG-64 } \\
\text { ILE-68 } \\
\text { PRO-72 } \\
\text { PHE-95 }\end{array}$ & $\begin{array}{l}\text { ASN-70 } \\
\text { GLY-123 }\end{array}$ & $\begin{array}{l}\text { ARG-64 } \\
\text { HIS-122 }\end{array}$ & - & - \\
\hline Ellagic acid & 5281855 & $-6.58 \pm 0.18$ & VAL-61 & $\begin{array}{l}\text { ARG-64 } \\
\text { ASN-70 } \\
\text { GLY-123 }\end{array}$ & $\begin{array}{l}\text { ARG-64 } \\
\text { HIS-122 }\end{array}$ & HIS-122 & ARG-64 \\
\hline Apigenin & 5280443 & $-6.18 \pm 0.15$ & $\begin{array}{l}\text { VAL-61 } \\
\text { THR-65 } \\
\text { ASN-70 } \\
\text { PRO-72 }\end{array}$ & $\begin{array}{l}\text { ARG-64 } \\
\text { LEU-121 }\end{array}$ & & & \\
\hline Rutin & 5280805 & $-6.30 \pm 0.24$ & $\begin{array}{l}\text { PHE-95 } \\
\text { TRP-99 }\end{array}$ & $\begin{array}{l}\text { ASN-70 } \\
\text { LEU-121 } \\
\text { GLY-123 }\end{array}$ & - & HIS-122 & $\begin{array}{l}\text { ARG-64 } \\
\text { HIS-122 }\end{array}$ \\
\hline Kaempferol & 5280863 & $-6.12 \pm 0.10$ & LYS-117 & $\begin{array}{l}\text { SER-71 } \\
\text { PRO-76 } \\
\text { ASP-118 } \\
\text { GLY-119 }\end{array}$ & - & - & - \\
\hline Quercetin & 5280343 & $-6.12 \pm 0.15$ & $\begin{array}{l}\text { PRO-69 } \\
\text { LYS-117 }\end{array}$ & $\begin{array}{l}\text { ASP-66 } \\
\text { SER-71 } \\
\text { ASP-118 } \\
\text { GLY-199 }\end{array}$ & & - & - \\
\hline
\end{tabular}

TNFL8 with the extracts of $X$. aethiopica [33]. X. aethiopica is a spice that has been used in orthodox medicine for the management of various diseases associated with a dysfunctional inflammatory response in various regions of West Africa [34]. The pharmacologically active compounds present in the fruit include L-pinocarveol, 13epimanoyl oxide, 4-terpineol, apigenin, caffeic acid, chlorogenic acid, cis- $\alpha$-copaene-8-ol, cumic alcohol, ellagic acid, eudesma-1,3-dien-11-ol, kaempferol, linoleic acid, linolenic acid, myrtenol, o-cymene, oleic acid, palmitic acid, palmitoleic acid, quercetin, rutin, stearic acid, xylopic acid, $\alpha$-pinene, $\alpha$-terpineol, 1,8-cineole, and $\beta$ pinene [33, 35, 36].

Different therapeutic compounds could induce distinctive pharmacodynamic responses based on variability in their binding modes and binding affinities. The binding-free energies reflect the respective binding affinity of the eight phytochemicals to the model. The results showed that the cytokine interacted favourably with the selected phytochemicals with ellagic acid having the highest binding affinity since it possesses the least binding-free energy followed by rutin and apigenin as presented in Table 2, while caffeic acid was the least among the eight phytochemicals with the binding energy of $-\mathrm{n} 5.22 \pm 0.26 \mathrm{kcal} / \mathrm{mol}$. This indicated the bioactive compounds from the fruit Xylopia aethiopica could impair the functionality of the cytokine as presented through the in vivo study. These phytochemicals have earlier been reported by different studies to downregulate many degenerative processes particularly those that are related to inflammatory responses [34, 37].

The prediction of binding sites and visualization of relevant non-covalent interactions in the $3 \mathrm{D}$ structures were carried out via the PLIP server to understand the biochemical functionality vis-à-vis the responses of the protein to drugs (Table 2, Fig. 10). Xylopic acid and quercetin formed common hydrophobic interaction patterns with PRO-69 and a hydrogen bond to ASP-66 via their carboxyl groups. All the selected ligands interacted with the TNFL8 through hydrophobic contacts and hydrogen bindings. Caffeic acid, chlorogenic acid, and ellagic acid formed salt bridges with some amino acid residues of the cytokine via their carboxyl groups. Individual interaction sub-patterns were identified among the ligands with one of the most unique interaction patterns revealed in the complex with ellagic acid and rutin, where analogous $\pi$-stacking with HIS- 122 and $\pi$-cation interactions with ARG-64 occurred. $\pi$-Stacking which indicates the non-covalent interactions between aromatic rings and $\pi$-cation interactions have long been known as major constituents of ligand-protein interfaces $[38,39]$. Furthermore, the interactions of the 


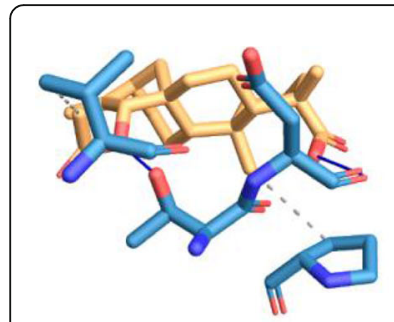

(a)

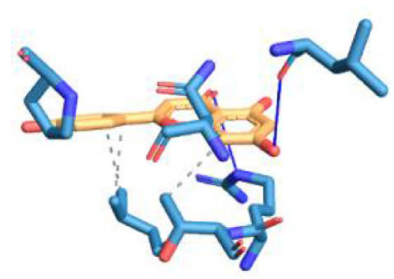

(e)

Keys:

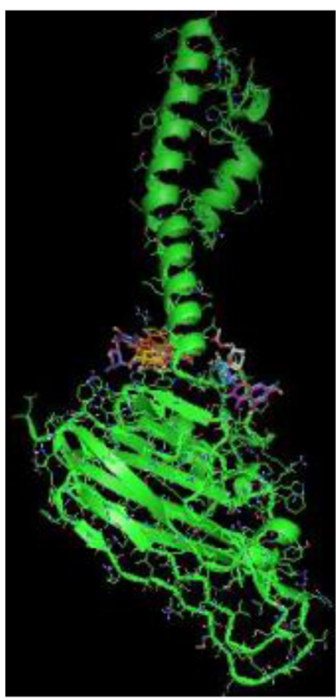

A

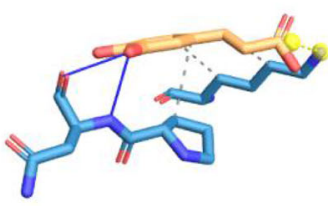

(b)

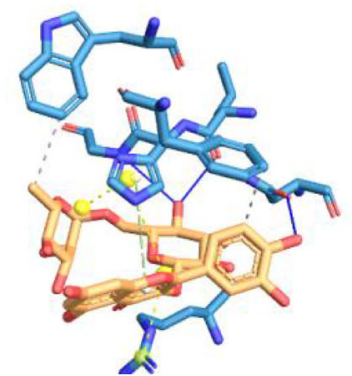

(f)

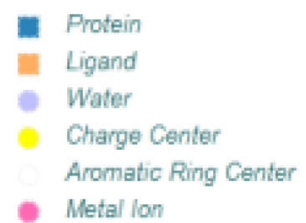

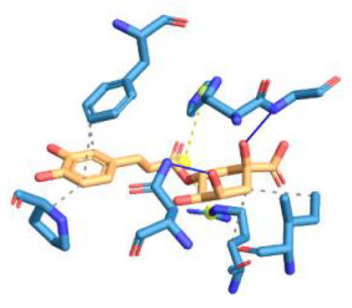

(c)

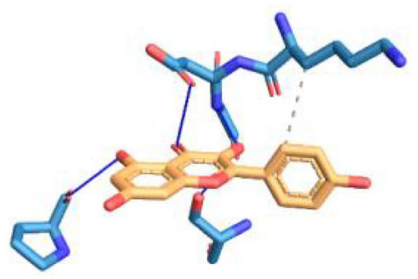

(g)

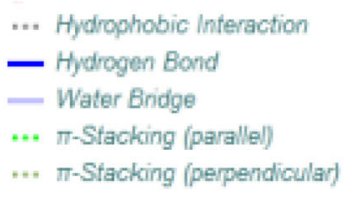

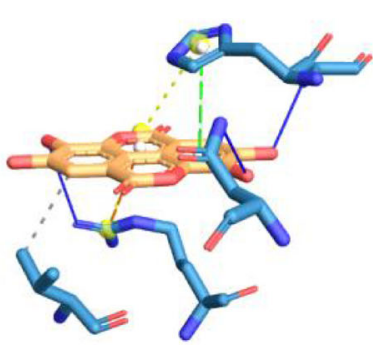

(d)

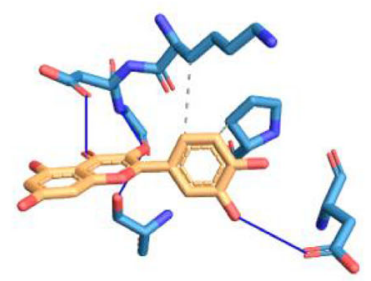

(h)

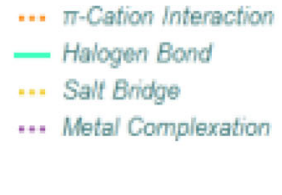

"." $\pi$-Cation Interaction

gogen Bond

.. Salt Eindge

Fig. 10 The identification of non-covalent interactions between TNFL8 and the selected ligands a xylopic acid, $\mathbf{b}$ caffeic acid, $\mathbf{c}$ chlorogenic acid, $\mathbf{d}$ ellagic acid, e apigenin, $\mathbf{f}$ rutin, $\mathbf{g}$ kaempferol, and $\mathbf{h}$ quercetin

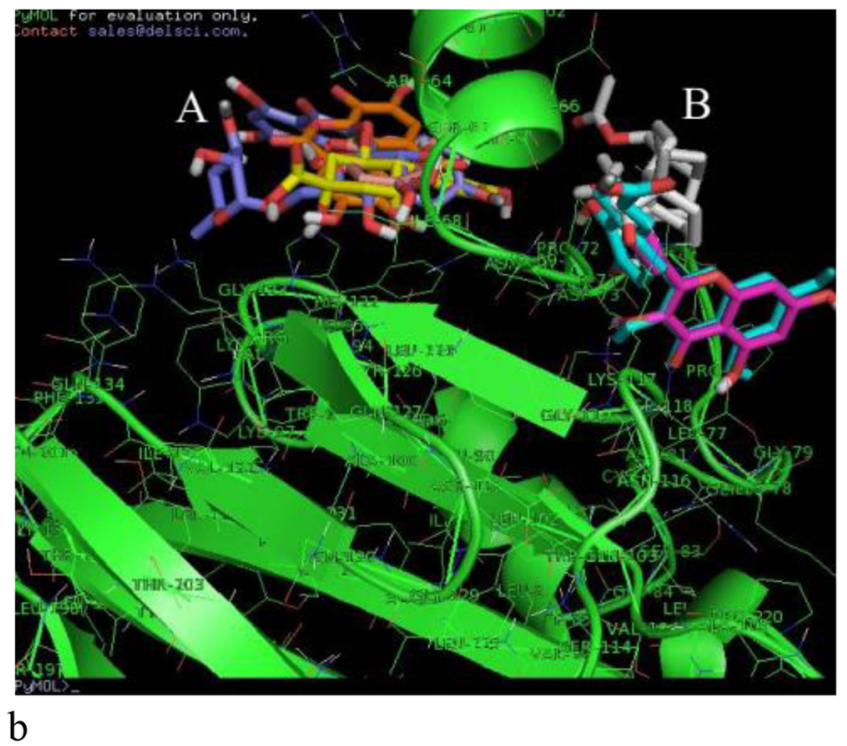

Fig. 11 Two binding pockets ( $\mathrm{A}$ and $\mathrm{B}$ ) for the phytochemicals $\mathbf{a}$ full and $\mathbf{b}$ close up 


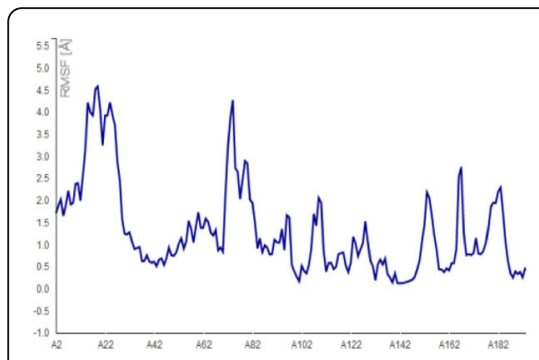

(a)

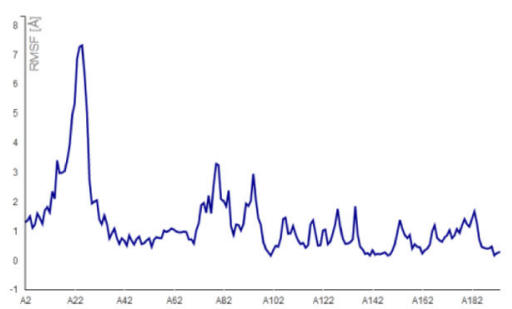

(d)

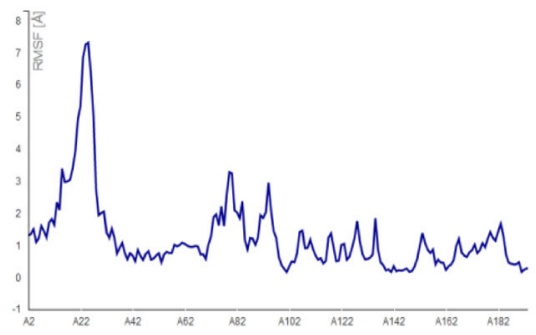

(g)

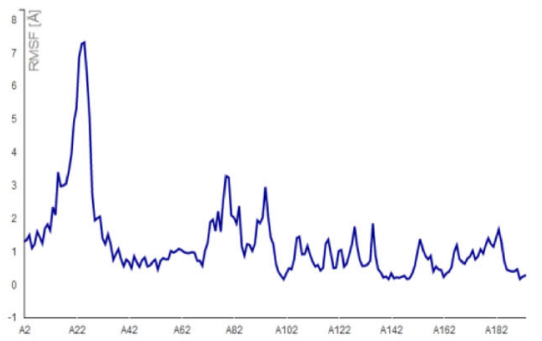

(b)

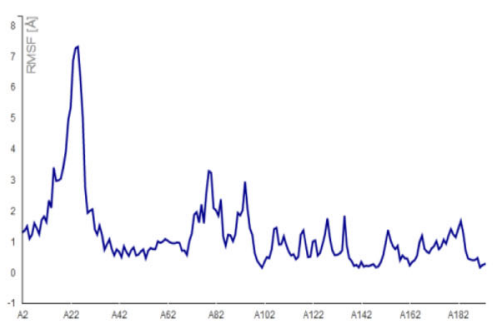

(e)

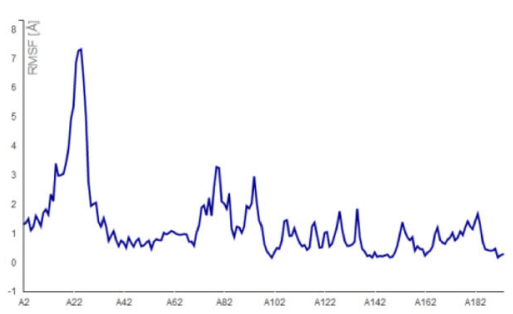

(h)

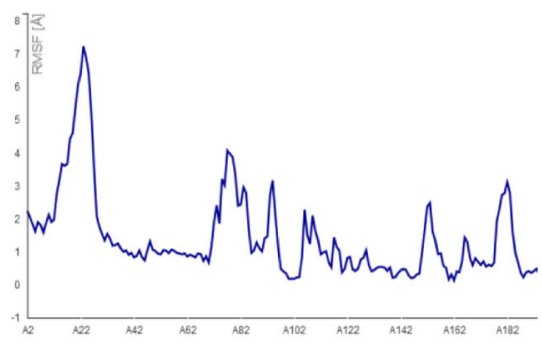

(c)

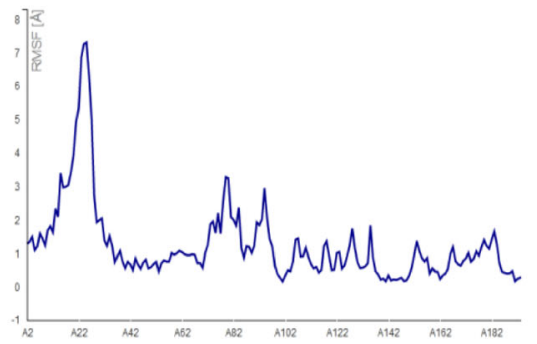

(f)

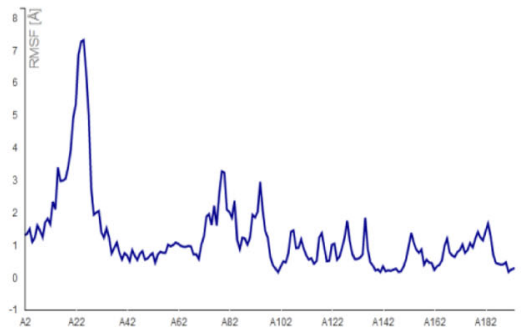

(i)

Fig. 12 Molecular dynamics simulation showing the RMSF of a the wild-type TNFL8 and the complexes between TNFL8 and $\mathbf{b}$ xylopic acid, $\mathbf{c}$ caffeic acid, $\mathbf{d}$ chlorogenic acid, e ellagic acid, $\mathbf{f}$ apigenin, $\mathbf{g}$ rutin, $\mathbf{h}$ kaempferol, and $\mathbf{i}$ quercetin

phytochemicals with the cytokine were found to occur in two binding pockets for the different ligands. The ligands found in pocket $\mathrm{A}$ are chlorogenic acid, apigenin, rutin, and ellagic acid, while those in pocket $\mathrm{B}$ are xylopic acid, caffeic acid, quercetin, and kaempferol (Fig. 11). This implies that the binding of phytochemicals could induce allosteric modulation which could thus regulate the physiological functions of the cytokine [40]. Common residues found in pocket A where chlorogenic acid, apigenin, rutin, and ellagic acid bound include ARG-64 and ASN-70 whereas PRO-69 was common in pocket B where xylopic acid, caffeic acid, quercetin, and kaempferol are present. This study indicates that the selected bioactive compounds most especially ellagic acid, apigenin, and rutin which demonstrated the highest docking interaction energies toward the cytokine could have sufficient specificities and potencies to bind and modulate the function of TNFL8. Moreover, ASN-70 and ARG-64 in pocket A to which the phytochemicals with lower binding energies anchored could be the key residues at the active site of the cytokine.
Notably, the interaction model proposed corroborated a previously available study on the inhibition of the expressions of splenic TNFRSF8 by ethanolic extract of $X$. aethiopica [37].

The flexibility of the cytokine and its complexes with the selected phytochemicals were simulated using an online tool (CABS-flex 2.0 server) [41]. The cytokinephytochemical interactions generated RMSF graphs were evaluated based on the RMSF. The protein structural simulation generated RMSF graphs showed the flexibility of the amino acids were relatively low for the complexes while compared to the wild-type TNFL8 without bound phytochemicals. Caffeic acid had relatively higher fluctuations compared to the other phytochemicals. The changes in the fluctuations of the residues at the pockets where the phytochemical binding occurred indicate the interactions of the selected phytochemicals at the active sites of TNFL 8 could enhance the rigidity of the amino acids in the active sites. Thus, the binding of the phytochemicals with TNFL8 could influence the TNFL8/ TNFRSF8 interactions and subsequence biologic 
functions and the innate capability to initiate cellular responses. This could corroborate the therapeutic actions of these compounds to disorders that are associated with inflammatory responses $[34,37]$.

\section{Conclusion}

The study provides an alternative to the experimental structure determination of TNFRSF8 through an array of independent algorithms. Besides, the results of the molecular docking analysis were consistent with the previously reported in vivo activity of an extract containing the selected phytochemicals on TNFL8 in Wistar rat model. The structural model could provide a detailed understanding of molecular processes useful in the development of therapeutics in inflammatory disorders. However, experimental techniques such as X-ray crystallography and nuclear magnetic resonance are recommended to corroborate the changes in structure and dynamics between the free and bound forms of TNFL8.

\footnotetext{
Abbreviations

I-TASSER: Iterative Threading ASSEmbly Refinement; TNFL8: Tumour necrosis factor ligand superfamily member 8; TNFRSF8: Tumour necrosis factor superfamily member 8; RMSF: The root-mean-square fluctuations; RMFR: Residues in most favoured regions; QMEAN: Qualitative Model Energy Analysis
}

\section{Acknowledgements}

Not applicable.

\section{Authors' contributions}

$\mathrm{BJO}, \mathrm{EBO}$, and ATO conceived and designed the study. BJO wrote the manuscript draft under the supervision of EBO and ATO. The authors read and approved the final manuscript.

\section{Funding}

Not applicable.

\section{Availability of data and materials}

Data and material are available upon request.

\section{Declarations}

Ethics approval and consent to participate

Not applicable.

\section{Consent for publication}

Not applicable.

\section{Competing interests}

The authors declare that they have no competing interests.

\begin{abstract}
Author details
${ }^{1}$ Department of Biochemistry, McPherson University, Seriki Sotayo, Ogun State, Nigeria. ${ }^{2}$ Department of Biochemistry, Ladoke Akintola University of Technology, Ogbomoso, Oyo State, Nigeria. ${ }^{3}$ Department of Biochemistry, University of Ilorin, Ilorin, Kwara State, Nigeria.
\end{abstract}

Received: 13 March 2021 Accepted: 18 May 2021

Published online: 05 June 2021

\section{References}

1. Wiley SR, Goodwin RG, Smith CA (1996) Reverse signaling via CD30 ligand. J Immunol 157(8):3635-3639
2. Nielson C, Fischer R, Fraga G, Aires D (2016) Loss of CD30 expression in anaplastic largecell lymphoma following brentuximab therapy. J Drugs Dermatol 15(7):894-895

3. Gottesman SR (2016) CD30: receptor, marker, target. Path Lab Med Int 8:27-36

4. Lee SY, Lee SY, Kandala G, Liou ML, Liou HC, Choi Y (1996) CD30/TNF receptor-associated factor interaction: NF-kappa B activation and binding specificity. Proc Natl Acad Sci USA 93(18):9699-9703. https://doi.org/10.1 073/pnas.93.18.9699

5. Shi JH, Sun SC (2018) Tumor necrosis factor receptor-associated factor regulation of nuclear factor $\mathrm{KB}$ and mitogen-activated protein kinase pathways. Front Immunol 9:1849. https://doi.org/10.3389/fimmu.2018.01849

6. Oflazoglu E, Grewal IS, Gerber H (2009) Targeting CD30/CD30L in oncology and autoimmune and inflammatory diseases. Adv Exp Med Biol 647:174185. https://doi.org/10.1007/978-0-387-89520-8_12

7. Sonar S, Lal G (2015) Role of tumor necrosis factor superfamily in neuroinflammation and autoimmunity. Front. Immunol. 6:364

8. Chiarle R, Podda A, Prolla G, Podack ER, Thorbecke GJ, Inghirami G (1999) CD30 overexpression enhances negative selection in the thymus and mediates programmed cell death via a Bcl-2-sensitive pathway. J Immunol 163(1):194-205

9. Hombach AA, Görgens A, Chmielewski M, Murke F, Kimpel J, Giebel B, Abken $H$ (2016) Superior therapeutic index in lymphoma therapy: CD30(+) CD34(+) hematopoietic stem cells resist a chimeric antigen receptor T-cell attack. Mol Ther. 24(8):1423-1434. https://doi.org/10.1038/mt.2016.82

10. Francisco JA, Cerveny CG, Meyer DL, Mixan BJ, Klussman K, Chace DF, Rejniak SX, Gordon KA, DeBlanc R, Toki BE, Law CL, Doronina SO, Siegall CB, Senter PD, Wahl AF (2003) CAC10-vcMMAE, an anti-CD30-monomethyl auristatin E conjugate with potent and selective antitumor activity. Blood. 102(4):1458-1465. https://doi.org/10.1182/blood-2003-01-0039

11. Deng C, Pan B, O'Connor OA (2013) Brentuximab vedotin. Clin Cancer Res 19(1):22-27. https://doi.org/10.1158/1078-0432.CCR-12-0290

12. Schmidt T, Bergner A, Schwede T (2014) Modelling three-dimensional protein structures for applications in drug design. Drug Dis Today 19(7): 890-987. https://doi.org/10.1016/j.drudis.2013.10.027

13. Goldfeld DA, Zhu K, Beuming T, Friesner RA (2013) Loop prediction for a GPCR homology model: algorithms and results. Proteins 81(2):214-228. https://doi.org/10.1002/prot.24178

14. Mehmood MA, Sehar U, Ahmad N (2014) Use of bioinformatics tools in different spheres of life sciences. J Data Mining Genom Proteom 5:158

15. Combet C, Blanchet C, Geourjon C, Deléage G (2000) NPS@: network protein sequence analysis. Trends Biochem Sci 25(3):147-150. https://doi. org/10.1016/S0968-0004(99)01540-6

16. Yang J, Zhang Y (2015) I-TASSER server: new development for protein structure and function predictions. Nucleic Acids Res 43(W1):W174-W181. https://doi.org/10.1093/nar/gkv342

17. Zhang C, Freddolino PL, Zhang Y (2017) COFACTOR: improved protein function prediction by combining structure, sequence and protein-protein interaction information. Nucleic Acids Res 45(W1):W291-W299. https://doi. org/10.1093/nar/gkx366

18. Ko J, Park H, Heo L, Seok C (2012) GalaxyWEB server for protein structure prediction and refinement. Nucleic Acids Res 40(W1):W294-W297. https:// doi.org/10.1093/nar/gks493

19. Laskowski RA, MacArthur MW, Moss DS, Thornton JM (1993) PROCHECK: a program to check the stereochemical quality of protein structures. J. Appl Crystal 26(2):283-291. https://doi.org/10.1107/S0021889892009944

20. Laskowski RA, Rullmannn JA, MacArthur MW, Kaptein R, Thornton JM (1996) AQUA and PROCHECK-NMR: programs for checking the quality of protein structures solved by NMR. J Biomol NMR 8(4):477-486. https://doi.org/10.1 007/BF00228148

21. Benkert P, Biasini M, Schwede T (2011) Toward the estimation of the absolute quality of individual protein structure models. Bioinformatics 27(3): 343-350. https://doi.org/10.1093/bioinformatics/btq662

22. Roy A, Yang J, Zhang Y (2012) COFACTOR: an accurate comparative algorithm for structure-based protein function annotation. Nucleic Acids Res 40(W1):W471-W477. https://doi.org/10.1093/nar/gks372

23. Kim S, Chen J, Cheng T, Gindulyte A, He J, He S, Li Q, Shoemaker BA, Thiessen PA, Yu B, Zaslavsky L, Zhang J, Bolton EE (2019) PubChem 2019 update: improved access to chemical data. Nucleic Acids Res 47(D1):D1102D1109. https://doi.org/10.1093/nar/gky1033

24. O'Boyle NM, Banck M, James CA, Morley C, Vandermeersch T, Hutchison GR (2011) Open Babel: an open chemical toolbox. J Cheminform 3(10):33. https://doi.org/10.1186/1758-2946-3-33 
25. Trott O, Olson AJ (2010) AutoDock Vina: improving the speed and accuracy of docking with a new scoring function, efficient optimization, and multithreading. J Comput Chem 31(2):455-461. https://doi.org/10.1002/ jcc. 21334

26. Salentin S, Schreiber S, Haupt VJ, Adasme MF, Schroeder M (2015) PLIP: fully automated protein-ligand interaction pro-filer. Nucleic Acids Res 43(W1): W443-W447. https://doi.org/10.1093/nar/gkv315

27. Kuriata A, Gierut AM, Oleniecki T, Ciemny MP, Kolinski A, Kurcinski M, Kmiecik S (2018) CABS-flex 2.0: a web server for fast simulations of flexibility of protein structures. Nucleic Acids Res 46(1):338-343

28. Mason JM, Arndt KM (2004) Coiled coil domains: stability, specificity and biological implications. ChemBioChem 5(2):170-176. https://doi.org/10.1002/ cbic.200300781

29. Benkert P, Tosatto SCE, Schomburg D (2008) QMEAN: a comprehensive scoring function for model quality assessment. Proteins 71(1):261-277. https://doi.org/10.1002/prot.21715

30. Fischer M, Harvima IT, Carvalho RFS, Möller C, Naukkarinen A, Enblad G, Nilsson G (2006) Mast cell CD30 ligand is upregulated in cutaneous inflammation and mediates degranulation-independent chemokine secretion. J Clin Invest 116(10):2748-2756. https://doi.org/10.1172/JCI24274

31. Gupta SC, Prasad S, Kim JH, Patchva S, Webb LJ, Priyadarsini IK, Aggarwal BB (2011) Multitargeting by curcumin as revealed by molecular interaction studies. Nat Prod Rep 28(12):1937-1955. https://doi.org/10.1039/c1np00051a

32. Basu A, Kurien BT, Tran H, Byrd B, Maher J, Schell J, Masek E, Barrett JR, Lyons TJ, Betts NM, Scofield RH (2018) Strawberries decrease circulating levels of tumor necrosis factor and lipid peroxides in obese adults with knee osteoarthritis. Food Funct 9(12):6218-6226. https://doi.org/10.1039/ C8FO01194J

33. Oso BJ, Boligon AA, Oladiji AT (2018) Metabolomic profiling of ethanolic extracts of the fruit of Xylopia aethiopica (Dunal) A. Rich using gas chromatography and high-performance liquid chromatography techniques. J Pharm Phytochem 7(1):2083-2090

34. Oso BJ, Oyewo EB, Oladiji AT (2017) Ethanolic, nhexane and aqueous partitioned extracts of Xylopia aethiopica fruit modulated inflammatory responses in turpentine oil induced acute inflammation in male Wistar rats. Int J Res Health Sci 5(2):1-10

35. Elhassan IA, Elamin EE, Ayoub SMH (2010) Chemical composition of essential oil in dried fruits of Xylopia aethiopica from Sudan. Open Access Med Arom Plants 1(1):24-28

36. Ezekwesili CN, Nwodo OFC, Eneh FU, Ogbunugafor HA (2010) Investigation of the chemical composition and biological activity of Xylopia aethiopica Dunal (Annonacae). Afr J Biotech 9(43):7352-7356

37. Oso BJ, Oyeleke OM, Oladiji AT (2018) Inhibition of the expressions of splenic TNF-alpha receptor superfamily 8, CD3 and CD20 by ethanolic extract of Xylopia aethiopica (Dunal) A. Rich Int J Bio Sci Appl 5(2):29-33

38. Wu R (2008) McMahon TB (2008) Investigation of cation-pi interactions in biological systems. J Am Chem Soc. 130(38):12554-12555. https://doi.org/1 $0.1021 / j a 802117 \mathrm{~s}$

39. Pyrkov TV, Pyrkova DV, Balitskaya ED, Efremov RG (2009) The role of stacking interactions in complexes of proteins with adenine and Guanine fragments of ligands. Acta Naturae 1(1):124-127. https://doi.org/10.32607/20758251-2 009-1-1-124-127

40. van Westen GJP, Gaulton A, Overington JP (2014) Chemical, target, and bioactive properties of allosteric modulation. PLoS Comput Biol 10:e1003559

41. Jamroz M, Kolinski A, Kmiecik S (2013) CABS-flex: server for fast simulation of protein structure fluctuations. Nucleic Acids Res 41(W1):W427-W431. https:// doi.org/10.1093/nar/gkt332

\section{Publisher's Note}

Springer Nature remains neutral with regard to jurisdictional claims in published maps and institutional affiliations.

\section{Submit your manuscript to a SpringerOpen ${ }^{\circ}$ journal and benefit from:}

- Convenient online submission

- Rigorous peer review

- Open access: articles freely available online

High visibility within the field

- Retaining the copyright to your article

Submit your next manuscript at $\boldsymbol{\nabla}$ springeropen.com 